\title{
Opinion Mining Research on Chinese Micro-blog
}

\author{
Changzhi Wang \\ China Electric Power Research Institute \\ Beijing, China \\ wangchangzhi@epri.sgcc.com.cn
}

Quanchao Liu

Beijing Engineering Applications Research Center of

High Volume Language Information Processing and

Cloud Computing, Beijing Institute of Technology

Beijing, China

\author{
Heyan Huang \\ Beijing Engineering Applications Research Center of \\ High Volume Language Information Processing and \\ Cloud Computing, Beijing Institute of Technology \\ Beijing, China \\ Xiaochuan Tian \\ State Grid Qingdao Power Supply Company \\ Shandong, China
}

\begin{abstract}
We propose to simultaneously identify opinion targets and opinion-bearing words based on multi-features in Chinese micro-blog post, and decompose our task into three phases: (1) construct opinion-bearing words dictionary and identify opinion-bearing word in a sentence from Chinese microblog; (2) design multiple features related to opinion target identification, containing Token, POS, WD, DDR; (3) design three kinds of different feature templates to identify featureopinion pairs <opinion target, opinion-bearing word $>$ in Chinese micro-blog post. The experiments show that our approach is very effective and get better performance than the baselines.
\end{abstract}

Keywords-Opinion mining, micro-blog, sentiment polarity, sentiment analysis

\section{INTRODUCTION}

In recent years, sentiment analysis, which mines opinions from information sources such as news, product reviews and twitter, has drawn much attention in the NLP field, such as [1], [2], [3], [4], [5] and so on, especially the micro-blog opinion mining. Micro-blog has become a very popular communication tool among internet users in China. According to the reports, the number of micro-blog users has increased from 63,110,000 by the end of 2010 to 274,000,000 in June 2012, and the number is still constantly growing. So many micro-blog users share opinions on different aspects of life everyday and express their various emotion and sentiment, such as joy, anger, grief, praise, criticism and so on, which makes people's opinion information expanded rapidly. As a result, it is very difficult to rely on the artificial method to mine opinions in micro-blog texts, there is an urgent requirement to help user analyse the massive information using computer.

[6] introduced Chinese micro-blog and we conclude that it mainly has three characteristics as following:

1) Due to the short text message, micro-blog has terms' sparsity, it is not suitable for opinion mining only based on terms.

2) There exist many homophonic words, abbreviated words, internet slang in micro-blog, such as " Bei Ju" standing for "tragedy”, "3Q” standing for "thanks”, “ Diao Si” standing for "young people who has no height, no wealth and no appearance" and so on.

Key Project of Chinese National Programs for Fundamental Research and Development (973 Program, No.2013CB329605)
3) There are a variety of relations between individual micro-blogs, including forwarding, commenting and sharing. It is very convenient for micro-blog users to express their opinions and sentiment.

The above characteristics of micro-blogs make it tough to extract opinion targets and opinion-bearing words. In order to further analyse opinions of micro-blogs, we propose a novel algorithm based on multi-features to simultaneously identify feature-opinion pairs <opinion target, opinion-bearing word>. The rest of this paper is structured as follows. In Sec. 2, we briefly summarize related works. Sec. 3 gives an overview for constructing opinion-bearing words dictionary and describing multiple features. The proposed approach is described in Sec. 4, followed by the experimental results in Sec. 5. Lastly, we conclude in Sec. 6.

\section{RELATED WORKS}

Although document-level/sentence-level sentiment analysis can provide the overall polarity of the whole text/sentence, it fails to detect the targets of the sentiment in texts/sentences.

For opinion mining of product reviews, properly identifying opinion targets and opinion-bearing words can help us construct domain-related entity thesaurus and sentiment vocabulary, as [7], [8] and [9] have described. In addition, if correctly identify the correspondence relation between opinion target and opinion-bearing words, we can generate visual product reviews. Opinion targets proposed by [10] are often nouns or noun phrases frequently mentioned, and they consider adjectives as candidates of opinion-bearing words. After extracting opinion targets, they first locate all the sentences containing opinion targets, and then take the adjectives modifying opinion targets as opinion-bearing words. However, the algorithm is further improved by [2], they filter out noun phrases not belonging to opinion targets and define ten kinds of syntactic relations, and then use them in the syntactic tree to extract opinion-bearing words.

Through linguistics and semantic analysis of review articles, [3] designed the rules to extract feature-opinion pairs <opinion target, opinion-bearing word $>$ for product reviews. [11] also proposed supervised learning algorithm to extract opinion targets and opinion-bearing words. They take this as a 
sequence labeling task, and use HMM to obtain the most likely tag sequence. Although the algorithm consider ordinal relation between sentences, it isn't good for fusion multi-features because HMM is a generative model.

[12] proposed a novel two-stage framework for mining opinion targets and opinion-bearing words, their method achieves superior performance over unsupervised methods. But they only considered adjectives as opinion-bearing words and ignored other type of words such as verbs or nouns. [13] proposed to separately identify opinion holders and targets with dependency parser in Chinese news texts. Their proposed approach shows better performance on opinion holder/target identification with the traditional Chinese test data, such as Chinese news.

However, in this paper we focus on the simultaneous identification of opinion targets and opinion-bearing words, and make full use of multi-features, combining with discriminative conditional random fields (CRF) model, to simultaneously extract opinion target and opinion-bearing words in a sentence. The input is a collection of micro-blogs and the output is feature-opinion pairs <opinion target, opinion-bearing word $>$. Our approach shows promising performance on simultaneous identification of opinion targets and opinion-bearing words, and the results are much better than the baseline results and most results reported in CCF Conference on Natural Language Processing \& Chinese Computing 2012\&2013 (NLP\&CC 2012\&2013).

\section{FEATURE EXTRACTIONS AND SELECTION}

\section{A. Data Preprocessing}

In our approach, rich features representations are used to simultaneously identify opinion targets and opinion-bearing words in Chinese micro-blog texts. In order to generate such features, much NLP work has to be done beforehand, such as micro-blogs normalization, word segmentation, POS tagging and so on.

In our experiment, 600 subjective micro-blog sentences labeled with "opinionated $=Y$ " are extracted from NLP\&CC 2012\&2013. In order to conveniently obtain rich features, we design a three-step approach for data preprocessing in this paper:

1) We build a simple rule-based model for micro-blog normalization which can correct simple spelling errors into normal form, such as “!!!!!” to “!” and “。 。 。 。” to “。”.

2) The URL and the parentheses enclosing only English words or numbers are all removed in sentences, and the emoticons are also deleted, such as “:-)”, “:-(”, “:D” and so on.

3) To enhance the precision of word segmentation in micro-blog texts, internet slang expressing opinions is first recognized with a new Chinese word segmentation tool ICTCLAS2013 ${ }^{1 .}$

\footnotetext{
${ }^{1}$ http://ictclas.nlpir.org/
}

\section{B. Opinion-bearing Words Dictionarys}

Opinion-bearing Word, a key indicator of an opinion, is the words or phrases containing polarity (i.e. positive or negative). Micro-blog users express their sentiment polarity towards opinion target with opinion-bearing words. According to the characteristics of micro-blog, the opinion-bearing words dictionary would be made up of two portions. One of them is provided by HowNet and National Taiwan University Sentiment Dictionary (NTUSD). After the removal of duplicated and unusual words, we obtain 11,036 negative and 7,019 positive opinion-bearing words. The other is internet slang appeared in social network. In order to obtain more abundant opinion-bearing words, especially for Chinese microblog, two persons from our lab would label internet slang used to express users' opinions in micro-blog texts. After the removal of some internet slang rarely being used and without explicit opinions, which come from National Language Resource Monitoring \& Research Center (Network Media), finally we achieve 848 opinion-bearing words with polarity, 791 of which have emotion tagging consistency. The data labeled by two persons is shown in Table 1 . " +1 " is positive opinion, while “- 1 " is negative.

TABLE I. THE DISTRIBUTION OF OPINION-BEARING WORDS WITH POLARITY

\begin{tabular}{lllll}
\hline \multicolumn{5}{c}{ Person 1 } \\
\hline & & +1 & -1 \\
Person 2 & +1 & $459(\mathrm{a})$ & $36(\mathrm{~b})$ & $495(\mathrm{a}+\mathrm{b})$ \\
& -1 & $21(\mathrm{c})$ & $332(\mathrm{~d})$ & $353(\mathrm{c}+\mathrm{d})$ \\
\hline
\end{tabular}

where $N=a+b+c+d$, and the meaning of each character is described as follows:

a represents the quantities that two persons have the same emotion tagging labeled “+1";

b represents the quantities that person 1 labels “- 1 ” but person 2 labels “+1";

c represents the quantities that person 1 labels “ +1 ” but person 2 labels “-1";

$\mathrm{d}$ represents the quantities that two persons have the same emotion tagging labeled “- 1 ”.

In particular, we focus on consistency check of emotion tagging by KAPPA value $\mathrm{K}$ :

$$
\begin{aligned}
K & =\frac{(a+d) / N-[(a+c)(a+b) / N+(b+d)(c+d) / N] / N}{1-[(a+c)(a+b) / N+(b+d)(c+d) / N] / N} \\
& =\frac{(459+332) / 848-[480 * 495 / 848+368 * 353 / 848] / 848}{1-[480 * 495 / 848+368 * 353 / 848] / 848} \\
& \approx 0.863
\end{aligned}
$$

The high KAPPA value means the reliable consistency of emotion tagging. Finally we keep the words with emotion tagging consistency and finally obtain 7,478 positive and 11,368 negative opinion-bearing words.

\section{Multi-features' Description}

In the following we will describe the features we employ as input for our CRF model. Opinion target, nouns or noun phrases usually governed by opinion-bearing words/phrases, is 
what the opinion is about. So we design multiple features to simultaneously identify opinion targets and opinion-bearing words in a sentence.

\section{(1) Token.}

This feature represents the string of the current token as a feature. Even though this feature is rather obvious, it can have considerable impact on the identification performance for opinion target and opinion-bearing word and should be a good indicator.

(2) POS.

This feature represents the part-of-speech tag of the current token as identified by the ICTCLAS2013. Opinion-bearing words are often adjectives or verbs and opinion targets are often nouns or noun phrases. At the same time, the CRF model is provided with additional information to identify opinion targets which are multiword expressions, i.e. noun combinations.

\section{(3) Word Distance (WD).}

[14] and [10] have shown that nouns/noun phrases are good candidates for opinion targets which are often closer to opinion-bearing word in a sentence, and opinion targets usually occur before or after the opinion-bearing word. So we take the word distance as Boolean feature. We will label the current token as 1 when the current token being closest to opinionbearing word is noun/noun phrase, otherwise the current token is labeled as 0 .

\section{(4) The Direct Dependency Relation (DDR).}

[15], [16] and [17] have successfully employed dependency relation in the dependency parse tree to link opinion expressions and the corresponding targets. All works identify direct dependency relations such as "amod" and "nsubj" as the most frequent and at the same time highly accurate connections between a target and an opinion expression in a sentence. So we label all tokens which have a direct dependency relation to opinion-bearing words in a sentence, such as "ATT (attribute)", "SBV (subject-verb)" and "VOB (verb-object)". ATT represents any adjectival phrase (opinion-bearing adjective) serves to modify the meaning of the nouns/noun phrases, SBV represents any noun/noun phrase is the nominative object of the verb (opinion-bearing verb) while VOB represents any noun/noun phrase is the accusative object of the verb. The HIT LTP is employed to get the direct dependency relation in a sentence, as shown in Fig.1. We label the current token having the "ATT", "SBV" and "VOB" direct dependency relation with opinion-bearing word as 1 , otherwise the current token is labeled as 0 .

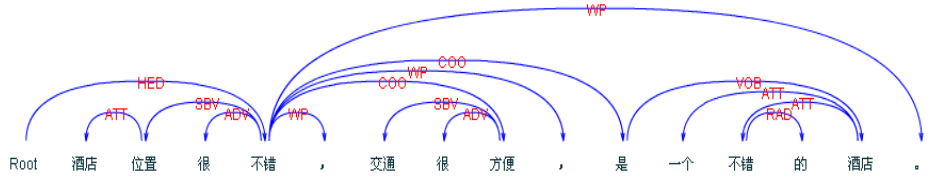

Fig. 1. The Direct Dependency Relation in The Sentence

\section{SIMULTANEOUSLY IDENTIFYING OPINION-BEARING WORDS AND OPINION TARGETS}

\section{A. $C R F$}

We simply label all the tokens in a sentence with the above four features. These features enable the CRF model to simultaneously identify opinion target and opinion-bearing words in a sentence. Our goal is to simultaneously identify opinion targets and opinion-bearing words from sentences which contain opinion targets and opinion-bearing words. This can be modeled as a sequence segmentation and labeling task. The CRF model receives a sequence of tokens $t_{1}, t_{2}, \cdots, t_{n}$ for which it has to predict a sequence of labels $l_{1}, l_{2}, \cdots, l_{n}$.

Generally speaking, the possible labels are represented following the IOB scheme: B-Target, identifying the beginning of a target, I-Target identifying the continuation of a target, and $\mathrm{O}$ for other (non-target) tokens. However, in our approach we design a novel scheme to label the token to be predicted in CRF, instead of the IOB scheme. The detailed is describes in Sec.6.

We model the sentences as a linear chain CRF which is based on an undirected graph. In the graph, each node corresponds to a token in the sentence and edges connect the adjacent tokens as they appear in the sentence. In our experiments, we use the CRF implementation from $\mathrm{CRF}++0.53^{1}$

\section{B. Preparing Feature Templates in CRF}

As CRF++ is designed as a general purpose tool, we have to specify the feature templates in advance. This file describes which features are used in training and testing. During our experiments, we design three kinds of templates to evaluate the simultaneous identification of opinion targets and opinionbearing words. In order to facilitate the description, we take the template T1 as example, which is used as the default template in $\mathrm{CRF}++$. The template $\mathrm{T} 1$ is shown in the Fig.4. The contents behind \# are discarded as comments, and other information can refer to the official web site ${ }^{2}$ for $\mathrm{CRF}++$.

$\begin{array}{ll}\text { \#Unigram } & \\ \text { \#Token } & \text { \#The previous token } \\ \text { U01:\%x[-1,0] } & \text { \#The current token } \\ \text { U02:\%x[0,0] } & \text { \#The next token } \\ \text { U03:\%x[1,0] } & \text { \#The previous token and the current token } \\ \text { U04:\%x[-1,0]/\%x[0,0] } & \text { \#The current token and the next token } \\ \text { U05:\%x[0,0]/\%x[1,0] } & \text { \#The POS of previous token } \\ \text { \#POS } & \text { \#The POS of current token } \\ \text { U11:\%x[-1,1] } & \text { \#The POS of next token } \\ \text { U13:\%x[1,1] } & \text { \#The POS of previous token and current } \\ \text { U14:\%x[-1,1]/\%x[0,1] } & \text { token } \\ \text { U15:\%x[0,1]/\%x[1,1] } & \text { \#The POS of current token and next token } \\ \text { \#WD } & \\ \text { \#DDR } & \\ \text { \#Bigram } & \\ \text { B }\end{array}$

Fig. 2. The Template T1

\footnotetext{
${ }^{1}$ http://code.google.com/p/crfpp/downloads/list

${ }^{2}$ http://crfpp.sourceforge.net/
} 
In order to contrast the experimental results under the condition of different templates, we additionally design templates T0 and T2. The contextual information appeared in the template $\mathrm{T} 1$ is removed, and only the current token information is retained in the template T0 as shown in Fig. 5. However, template T2 appends some combination information of multiple features on the basis of template $\mathrm{T} 1$, as shown in Fig. 6, namely that the template $\mathrm{T} 2$ contains not only the information of $\mathrm{T} 1$ but also the combination information of multiple features.

\begin{tabular}{|c|c|}
\hline \#Unigram & \\
\hline \#Token & \\
\hline $\mathrm{U} 02: \% x[0,0]$ & \#The current token \\
\hline \#POS & \\
\hline $\mathrm{U} 12: \% x[0,1]$ & \#The POS of current token \\
\hline \#WD & \\
\hline $\mathrm{U} 22: \% x[0,2]$ & \#The WD information of current token \\
\hline \#DDR & \\
\hline U32:\%x[0,3] & \#The DDR information of current token \\
\hline \#Bigram & \\
\hline $\mathrm{B}$ & \\
\hline
\end{tabular}

Fig. 3. The Template T0

$\begin{array}{ll}\text { \#Unigram } & \\ \text { \#Token+POS+WD } & \\ \text { U51:\%x[0,0]/\%x[0,1]/\%x[0,2] } & \begin{array}{l}\text { \#Combining the information of } \\ \text { token, POS and WD }\end{array} \\ \begin{array}{ll}\text { \#Token+POS+WD+DDR } & \\ \text { U61:\%x[0,0]/\%x[0,1]/\%x[0,2]/ } & \text { \#Combining the information of } \\ \%[0,3] & \text { token, POS, WD and DDR } \\ \text { \#Bigram } & \\ \text { B } & \end{array}\end{array}$

Fig. 4. The combination information of multiple features on the basis of template $\mathrm{T} 1$

\section{EXPERIMENT}

In this study, we consider an opinion-bearing word is a key indicator of an opinion. Therefore, we first identify opinionbearing words from a given sentence and then label the corresponding opinion targets based on multi-features described in Sec. 3.3.

\section{A. Data Sets and Experiments Settings}

The labeled data sets in NLP\&CC 2012\&2013, a total of 405 micro-blogs, are provided by Tencent Weibo, including four topics: hui_rong_an, ipad, kang_ri_shen_ju_sample and ke_bi_sample. We reserve the sentences labeled with "opinionated=Y" in a micro-blog, and then use opinion-bearing words and target_word (opinion target) to generate featureopinion pairs <opinion target, opinion-bearing word> for our evaluation. There are altogether 600 subjective sentences in data sets, and if there have several subjective sentences in a micro-blog, we firstly extract feature-opinion pairs from the current sentence. When there is no opinion target found in the current sentence, we are looking ahead until the first sentence. If no opinion target is found, we are looking backward from the current sentence until the last sentence.

In the process of training and testing, these data sets are processed into the format required by the $\mathrm{CRF}++$. According to the descriptions in Sec.3.3, each token has five columns, followed by the token itself, POS, WD, DDR and manual annotation category information in that order. Multiple sequences of tokens form a sentence, and these sentences are separated by a blank line. We design the following scheme for manual annotation category information, as shown in Table 2. When two (or more) adjacent tags are the same in a sentence, we combine the tags as one.

\section{TABLE II. ThE TAg SETS AND DESCRIPTION IN CRF++ TEMPLATE}

\begin{tabular}{|l|l|}
\hline \multicolumn{1}{|c|}{ Tag sets } & \multicolumn{1}{c|}{ Description } \\
\hline OT & Opinion target \\
\hline OW & Opinion-bearing word \\
\hline BG & Other background word \\
\hline
\end{tabular}

In order to avoid over-fitting or under-fitting, we adopt 10fold cross-validation in the experiments. Namely data sets would be randomly divided into 10 parts, 9 parts of them are used as training sets and the others are used to test. We repeat the process for 10 times and finally take the average value. Furthermore, we divide 600 subjective sentences from the labeled data sets into different data size, and respectively use a 10 -fold cross-validation so that we can observe the performance under the conditions of different data size. In this experiment, we adopt the default parameters in $\mathrm{CRF}++0.53$ and the window size of Token and POS is set to 3 separately while the window size of WD and DDR is set to 1 , namely WD and DDR use the current window.

\section{B. Performance Evaluation Method}

Performance evaluation is strictly matching in phrase-level, namely only when both opinion target and opinion-bearing word are correctly identified in a sentence, we think that the simultaneous identification of feature-opinion pairs <opinion target, opinion-bearing word $>$ is successful. We adopt Precision (P), Recall (R) and F-measure (F) to evaluate the algorithm performance.

$$
\begin{gathered}
\text { Pr ecision }=\frac{\text { Number of correct identified feature }- \text { opinion pairs }}{\text { Number of all identified feature }- \text { opinion pairs }} \\
\text { Recall }=\frac{\text { Number of correct identified feature }- \text { opinion pairs }}{\text { Number of all correct feature }- \text { opinion pairs }} \\
\qquad F-\text { measure }=\frac{2 * \text { Pr ecision } * \text { Recall }}{\text { Pr ecision }+ \text { Recall }}
\end{gathered}
$$

\section{Performance Comparison}

We first establish a baseline system, and reference to the views proposed by [10], [10] thinks that the POS is an important basis for judging sentiment information. So we only consider the token itself and the POS as basic features in the baseline system. Finally, we take template T1 as the default template and have a 10-fold cross-validation under the conditions of different data size. The results are shown in the following table.

TABLE III. THE RESULTS OF BASELINE SYSTEM

\begin{tabular}{|l|l|l|l|l|}
\hline \multirow{2}{*}{ Basic features } & \multirow{2}{*}{ Data size } & \multicolumn{3}{|c|}{ Results } \\
\cline { 3 - 5 } & & $\mathbf{P ( \% )}$ & $\mathbf{R}(\%)$ & $\mathbf{F ( \% )}$ \\
\hline \multirow{4}{*}{ Token+POS } & 100 & 68.7 & 61.0 & 64.6 \\
\cline { 2 - 5 } & 200 & 72.7 & 63.4 & 67.7 \\
\cline { 2 - 5 } & 400 & 72.6 & 69.9 & 71.2 \\
\cline { 2 - 5 } & 600 & 75.5 & 72.2 & 73.8 \\
\hline
\end{tabular}


From table 3 we know the overall performance is constantly increasing when the data size increases, and the precision is higher than the recall, which indicates there are many missing feature-opinion pairs in the process of identification. So we need more features, as illustrated in table 4 , to process 600 subjective sentences using the default template T1.

TABLE IV. THE IDENTIFICATION RESULTS BASED ON MULTI-FEATURES

\begin{tabular}{|l|l|l|l|}
\hline \multirow{2}{*}{\multicolumn{1}{|c|}{ Features }} & \multicolumn{3}{|c|}{ Results } \\
\cline { 2 - 4 } & $\mathbf{P ( \% )}$ & $\mathbf{R ( \% )}$ & $\mathbf{F ( \% )}$ \\
\hline Token+POS & 75.5 & 72.2 & 73.8 \\
\hline Token+POS+WD & 75.9 & 73.1 & 74.5 \\
\hline Token+POS+WD+DDR & 77.3 & 80.1 & 78.7 \\
\hline
\end{tabular}

On the basis of basic features, we add WD and DDR into our approach. From table 4 we know WD and DDR play an important role in identifying feature-opinion pairs <opinion target, opinion-bearing word $>$. The experimental results show that adding WD and DDR can effectively help our approach improve the performance of simultaneously identifying opinion target and opinion-bearing words. Especially using DDR feature makes our approach fully consider the dependency relation between opinion target and opinion-bearing word in the process of training. At the same time, the generated model would make more accurate prediction to some extent.

However, the above experiments utilize the feature template $\mathrm{T} 1$ and ignore the combination information of multiple features in CRF model. So we design three kinds of feature template to verify the effects on feature-opinion pairs, and use four features to do the experiment under the conditions of different data size. The experimental results are shown in Fig.5.

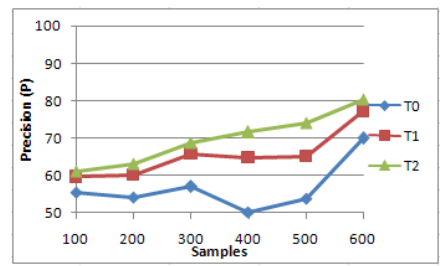

(a) Precision

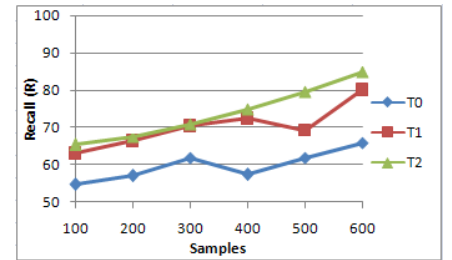

(b) Recall

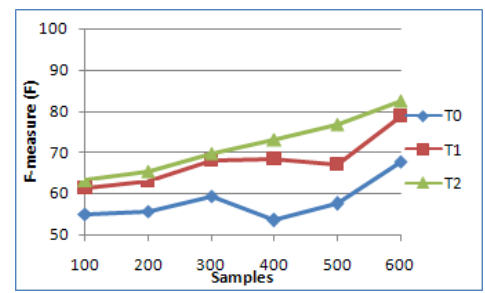

(c) F-measure

Fig. 5. The impact of using different templates and data size

The results from Fig. 5 show that both templates T2 and T1 are essentially the same in recall, but the precision of $\mathrm{T} 2$ is slightly ahead of T1. However, the performance of $\mathrm{T} 0$ is the worst because of no considering the context information. In the whole designing process of T1 and T2, we consider the context information of the word. Because the context category label of the word plays a very important role for the judgment of category label of the target word. With the continuous improvement of the templates, the experimental results are relatively better. Especially using the combination information of multiple features of template T2 achieves the best effect on identifying feature-opinion pairs <opinion target, opinionbearing word>. So the experiment achieves the best results using above four features and template $\mathrm{T} 2$.

Furthermore, the algorithm in [18] was applied to our data and template T2, the result is not so good as ours. The comparison is shown in table 5.

TABLE V. THE COMPARISON RESULTS

\begin{tabular}{|l|l|l|l|}
\hline \multirow{2}{*}{ Identification method } & \multicolumn{3}{|c|}{ Results } \\
\cline { 2 - 4 } & $\mathbf{P ( \% )}$ & $\mathbf{R}(\%)$ & $\mathbf{F ( \% )}$ \\
\hline The algorithm in [18] & 77.9 & 83.5 & 80.6 \\
\hline Multi-features identification & 80.5 & 84.9 & 82.6 \\
\hline
\end{tabular}

The algorithm in [18] only considers Token, POS and Semantic Role Labeling (SRL), which maybe lose some opinion target information because of features' sparsity of Chinese micro-blog. As shown in Table 5, without WD and DDR features in [18], the F-measure of the algorithm is $80.6 \%$, which is still lower than our $82.6 \%$. The experimental results show that WD and DDR do help identify opinion target. During the process of identification, Token, POS, WD, DDR and SRL all play important roles, and we will compare them using CRF model in future.

From Table 5 we can also see that the combination of multiple features contributes to the overall improvement. In addition, according to opinion-bearing word we can obtain the sentiment polarity of opinion target, and through NLP\&CC 2012\&2013's test data, our approach provides better performance than most systems reported at NLP\&CC 2012\&2013.

\section{CONCLUSIONS AND FUTURE WORKS}

In this paper, we investigate the problem of simultaneously identifying opinion targets and opinion-bearing words in opinionated sentences of Chinese micro-blog based on multifeatures. Our proposed approach shows good performance through the experiments. In future the work will focus on the following two aspects:

1) Compare Token, POS, WD, DDR and SRL in CRF model, and find out which has the better performance during the process of identifying opinion targets and opinion-bearing words.

2) There are more relations between individual micro-blogs, such as forwarding, commenting, sharing and so on. We need to take the relations into consideration for opinion mining.

\section{ACKNOWLEDGMENT}

We would like to thank the anonymous reviewers for many valuable comments and helpful suggestions. This paper is financially supported by Key Project of Chinese National Programs for Fundamental Research and Development (973 Program, No.2013CB329605). 


\section{REFERENCES}

[1] Minqing Hu and Bing Liu. 2004. Mining Opinion Features in Customer Reviews. In Proc. of AAAI-04.

[2] Popescu A M, Etzioni O. Extracting product features and opinions from reviews[M]//Natural language processing and text mining. Springer London, 2007: 9-28.

[3] Kamal A, Abulaish M, Anwar T. Mining feature-opinion pairs and their reliability scores from web opinion sources[C]/Proceedings of the 2nd International Conference on Web Intelligence, Mining and Semantics. ACM, 2012: 15.

[4] Gokulakrishnan B, Priyanthan P, Ragavan T, et al. Opinion mining and sentiment analysis on a Twitter data stream[C]//Advances in ICT for Emerging Regions (ICTer), 2012 International Conference on. IEEE, 2012: 182-188.

[5] Yang B, Cardie C. Joint inference for fine-grained opinion extraction[C]//Proceedings of ACL. 2013.

[6] Liu Q, Feng C, Huang H. Emotional Tendency Identification for Microblog Topics Based on Multiple Characteristics[J]. 2012.

[7] Kanayama H, Nasukawa T. Fully automatic lexicon expansion for domain-oriented sentiment analysis[C]/Proceedings of the 2006 Conference on Empirical Methods in Natural Language Processing. Association for Computational Linguistics, 2006: 355-363.

[8] Kaji N, Kitsuregawa M. Building lexicon for sentiment analysis from massive collection of HTML documents[C]//Proceedings of the joint conference on empirical methods in natural language processing and computational natural language learning (EMNLP-CoNLL). 2007: 10751083.

[9] Qiu G, Liu B, Bu J, et al. Expanding domain sentiment lexicon through double propagation[C]//Proceedings of the 21st international jont conference on Artifical intelligence. 2009: 1199-1204.
[10] $\mathrm{Hu} \mathrm{M}$, Liu B. Mining and summarizing customer reviews[C]//Proceedings of the tenth ACM SIGKDD international conference on Knowledge discovery and data mining. ACM, 2004: 168177.

[11] Jin W, Ho H H, Srihari R K. OpinionMiner: a novel machine learning system for web opinion mining and extraction[C]//Proceedings of the 15th ACM SIGKDD international conference on Knowledge discovery and data mining. ACM, 2009: 1195-1204.

[12] Xu L, Liu K, Lai S, et al. Mining Opinion Words and Opinion Targets in a Two-Stage Framework[J]. 2013.

[13] Lu B. Identifying opinion holders and targets with dependency parser in Chinese news texts[C]//Proceedings of the NAACL HLT 2010 Student Research Workshop. Association for Computational Linguistics, 2010: 46-51.

[14] Yi J, Nasukawa T, Bunescu R, et al. Sentiment analyzer: Extracting sentiments about a given topic using natural language processing techniques[C]//Data Mining, 2003. ICDM 2003. Third IEEE International Conference on. IEEE, 2003: 427-434.

[15] Zhuang L, Jing F, Zhu $\mathrm{X}$ Y. Movie review mining and summarization[C]//Proceedings of the 15th ACM international conference on Information and knowledge management. ACM, 2006: 43-50.

[16] Kessler J S, Nicolov N. Targeting Sentiment Expressions through Supervised Ranking of Linguistic Configurations[C]//ICWSM. 2009.

[17] Del Corro L, Gemulla R. ClausIE: clause-based open information extraction[C]//Proceedings of the 22nd international conference on World Wide Web. International World Wide Web Conferences Steering Committee, 2013: 355-366.

[18] Shi H. Research on fine-grained sentiment analysis[D]. Soochow University,
2013:
46-49. 